\title{
Efeitos sobre a escolaridade no Brasil: um estudo com dados em painel no período
}

\section{2-2014}

\author{
Effects on education in Brazil: a study with panel data in the period 2002-2014 \\ Efectos sobre la educación en Brasil: un estudio con datos de panel en el período 2002-2014
}

Recebido: 27/09/2021 | Revisado: 30/09/2021 | Aceito: 02/10/2021 | Publicado: 03/10/2021

Tito Belchior Silva Moreira

ORCID: https://orcid.org/0000-0002-2382-1480 Universidade Católica de Brasília, Brazil

E-mail: titoeco@yahoo.com.br

Lacerda Sipriano Elias

ORCID: https://orcid.org/0000-0002-4484-2950 Universidade Católica de Brasília, Brazil

E-mail: lacerdastones@gmail.com

\begin{abstract}
Resumo
Este artigo avalia, empiricamente, efeitos sobre a educação no Brasil. O estudo aborda o assunto com dados em painel em efeito fixo e efeito aleatório por unidades da federação no período 2002-2014. Discute-se, utilizando outras variáveis explicativas, o impacto das despesas com esporte e lazer sobre a escolaridade. Com base nos dois diferentes modelos em painel, os resultados atestam a hipótese de que aumentos nas despesas em esporte e lazer produzem elevações nos anos de estudo. As variáveis que representam a despesa com saúde e saneamento, acesso à água potável, acesso à instalação adequada de esgoto, frequência escolar e desigualdade de renda são, também, estatisticamente significantes no estudo e confirmam os sinais esperados.
\end{abstract}

Palavras-chave: Escolaridade; Despesa pública; Dados em painel.

\begin{abstract}
This article empirically assesses effects on education in Brazil. The study addresses the issue with panel data in fixed effect and random effect by units of the federation in the period 2002-2014. Using other explanatory variables, the impact of spending on sport and leisure on education is discussed. Based on the two different panel models, the results support the hypothesis that increases in spending on sport and leisure produce increases in the years of study. The variables that represent expenditure on health and sanitation, access to drinking water, access to adequate sewage installation, school attendance and income inequality are also statistically significant in the study and confirm the expected signs.
\end{abstract}

Keywords: Education; Public expense; Panel data.

\section{Resumen}

Este artículo evalúa empíricamente los efectos sobre la educación en Brasil. El estudio aborda el tema de los datos de panel en efecto fijo y efecto aleatório por unidades de la federación en el período 2002-2014. Utilizando otras variables explicativas, se discute el impacto del gasto en deportes y ocio en la educación. A partir de los dos modelos de panel diferentes, los resultados apoyan la hipótesis de que los incrementos en el gasto en deporte y ocio producen incrementos en los años de estudio. Las variables que representan gasto en salud y saneamiento, acceso a agua potable, acceso a alcantarillado adecuado, asistencia escolar y desigualdad de ingresos también son estadísticamente significativas en el estudio y confirman los signos esperados.

Palabras llave: Educación; Gasto público; Panel de datos.

\section{Introdução}

Elevar indicadores educacionais é fator fundamental para objetivar o alcance de metas de desenvolvimento humano e econômico, redução de desigualdades, melhorias em saúde e saneamento, geração de renda e qualificação do mercado de trabalho, contudo as políticas setoriais precisam estar integradas para obter sustentabilidade.

De acordo com dados do Instituto de Pesquisa Econômica e Aplicada (IPEA), o Brasil registrou, em 2002, em um dos índices de escolaridade, uma média de 6,1 anos de estudo alcançados por pessoas com 25 anos ou mais de idade, contudo esse valor veio aumentando ao longo dos anos e registrou 7,7 anos em 2013 e 7,8 em 2014. 
No entanto, políticas públicas voltadas para o aumento dos níveis educacionais, redução das defasagens e evasões escolares, além de ações para fortalecer a frequência escolar, a motivação e a aprendizagem são fundamentais para reduzir desigualdades e, sobretudo, qualificar talentos e gerar bem estar para famílias e sociedade.

A escola é o ambiente transformador de vidas e que abre caminhos para realizar sonhos, é o local onde os debates são construídos coletivamente com abordagem crítica construtiva e, nesse sentido, precisam de investimentos em todos os setores que promovam a aprendizagem e a melhoria dos índices educacionais.

Silva (2015) destaca que a inserção social do indivíduo é potencializada pela educação, ou seja, é observada a conquista ao direito fundamental do cidadão pleno e, por conseguinte, a redução das desigualdades.

Feistel e Maestrelli (2012) apontam para a necessidade da existência de ambientes que favoreçam a prática coletiva e a permuta de informações entre as diversas áreas do conhecimento voltadas para a construção contextualizada e significativa da aprendizagem.

Todavia, para elaborar planejamentos e estipular metas para o alcance dos índices educacionais, estudos sobre o momento atual e os obstáculos observados são, fundamentalmente, importantes.

Moraes, Souza e Amparo (2009) argumentam que os insucessos verificados na área educacional solicitam reflexões das questões relacionadas às desigualdades educacionais em conjunto com as características sociais.

Para Neri (2008), a agenda de políticas públicas tem apresentado pouco avanço no setor de saneamento básico e a falta de esgoto tratado que atinge grande parte da população no Brasil, afeta as mais variadas áreas, inclusive a educação.

Essa pesquisa discute efeitos sobre a escolaridade no Brasil, no período 2002-2014, utilizando dados em painel com efeito fixo e efeito aleatório por unidades da federação. As variáveis explicativas utilizadas no estudo são o coeficiente de Gini, a frequência escolar das pessoas na faixa etária dos 7 aos 14 anos de idade, as despesas em saúde e saneamento, o acesso à água potável, o acesso à rede adequada de esgoto e a variável de interesse, isto é, as despesas em esporte e lazer, que fundamenta nossa hipótese, pois acreditamos evidenciar uma relação direta com a taxa de escolaridade, isto é anos de estudo da população de 25 anos ou mais de idade.

O trabalho está distribuído em cinco seções. Além dessa introdução, a revisão da literatura é apresentada na seção 2, a metodologia, que inclui base de dados e modelos econométricos, está na seção 3, resultados e discussão na seção 4 e, na seção 5 são apresentadas as considerações finais.

\section{Revisão da Literatura}

Melhorar os indicadores educacionais requer a coleta e análise de dados de diversos aspectos socioeconômicos, a pesquisa de sucessos observados na literatura e nas experiências construídas, além de inúmeros debates coletivos, com participação da sociedade, para desenhar e implementar políticas públicas integradas de planejamento, ações e investimentos que promovam o êxito nas propostas e oferta de serviços públicos eficazes, eficientes e efetivos.

Neri, Melo, Sacramento, Neri, Calcada, Scharth e Moreira (2009) argumentam que durante o século XX o Brasil se tornou um país predominantemente urbano, contudo essa aglomeração populacional apresenta custos das deseconomias, ademais, as economias de escalas observadas poderiam ter promovido a oferta de maior quantidade e melhores condições de serviços públicos, inclusive os associados ao saneamento básico.

Varela, Noronha Filho, Mesquita, Sousa, Souza e Santos (2020) afirmam que os itens que compõem o saneamento básico necessitam ser realizados de forma conjunta para beneficiar a população, que precisa participar do processo para conservar a natureza e a eficiência das ações coletivas em busca da sustentabilidade. 
Carvalho (2018) argumenta que a existência de disparidades entre campo e cidade nas regiões do Brasil reflete os diferentes cenários reais das escolas no país em função do acesso ao saneamento e, ainda, em relação à inacessibilidade desse serviço, os impactos se apresentam na saúde, educação e na desigualdade das realidades vividas .

Segundo Scriptore (2016), as doenças associadas às condições precárias de saneamento básico prejudicam o rendimento escolar das crianças e assim são importantes para explicar indicadores educacionais. Sousa e Leite Filho (2008) apontam que políticas públicas inclusivas que fortaleçam as condições de saúde e bem estar da sociedade são fundamentais para produzir aumentos na escolaridade.

Scriptore, Azzoni e Menezes Filho (2018) utilizam dados municipais e dados em painel para observar o impacto positivo do saneamento básico sobre a educação, sobretudo verificam esse efeito nas residências da população de 6 a 14 anos de idade, que possuem banheiro e acesso à água canalizada com a utilização da rede de distribuição.

Segundo Kassouf (1995), a ausência do abastecimento de água, a inacessibilidade ao saneamento básico e reduzidos níveis educacionais potencializam os riscos à saúde, pois os cenários domésticos nessas condições podem propiciar o surgimento de tais evidências.

Carli e Costa (2020) apontam para a preocupação quanto à concretização do direito à água potável e ao saneamento básico, em função da exaustão da natureza e dos agentes poluentes.

Silva e Esperidião (2017) analisam os impactos do saneamento básico e desenvolvimento econômico da região nordeste do Brasil e constatam que o acesso à água e esgotamento sanitário produzem impactos diretos sobre as questões socioeconômicas, e as localidades com maiores evidências de melhoria da educação apontam para maiores demandas por serviços de saneamento básico.

Gandra, Vieira, Rodrigues e Oliveira (2016) mostram que, em média, o atraso escolar tem menor chance de ocorrência em populações que recebem serviços de saneamento básico em suas moradias, assim, o acesso a serviços de abastecimento de água e coleta de esgoto proporcionam a redução desse indicador educacional.

Prearo, Maraccini e do Carmo Romeiro (2015) constatam que o Índice de Desenvolvimento Humano Municipal sofre algum efeito, estatisticamente significativo, das variáveis despesas com educação, saneamento básico, assistência social e urbanismo, o que pode sugerir que os municípios necessitam direcionar à população, as atenções iniciais, para então, buscarem o crescimento dos demais setores.

Junior e Silva (2019), estudando a escolaridade na região sul do Brasil com dados em painel em dois biênios 2004/2005 e 2014/2015, verificam que a renda mínima e água encanada são fatores com impactos positivos no primeiro período, sobre esse indicador educacional, e o índice de renda ainda se manteve igual na análise do segundo período.

Borchers e Meiners (2019), em modelo econométrico para analisar concentração de renda e educação, utilizam testes que mostram, mesmo considerando as diferenças regionais, que os impactos de um ano a mais de estudo são, de forma igual, significativos em todas as regiões estudadas e, ademais, o acesso à educação é o grande desafio para o país enfrentar a desconcentração de renda.

Medeiros, Barbosa e Carvalhaes (2019) destacam a existência de uma limitação para o alcance dos efeitos da educação, mesmo no longo prazo, sobre a distribuição de renda, em função de outros fatores evidenciados, tais como a necessidade de reformas educacionais expressivas ou ainda a expansão da economia.

Abreu Souza, Taques, Oliveira e Alencar (2013), em estimativa com dados em painel no período 1995-2009, observam a importância da educação para a compreensão da desigualdade de renda. Duarte e Belmiro (2018), em estudo com dados em painel no período 1995-2014, encontram resultado estatisticamente significativo indicando que variáveis que retratam renda e educação tem relações inversas com o coeficiente de Gini. 
Kroth e Gonçalves (2019), em análise dos impactos das despesas públicas sobre a qualidade da educação, mostram que esses gastos contribuem de forma positiva para esse indicador, embora, em função do baixo coeficiente encontrado, podem sugerir que o volume de recursos aplicados não é suficiente para potencializar a qualidade, ou ainda, outa explicação é a má gestão dos recursos, contudo os gastos sociais podem ser complementares para o alcance da qualidade educacional.

Santos (2019) argumenta que os municípios têm reservados espaços para implementar políticas de esporte e lazer, mesmo enfrentando uma rigidez orçamentária e, no geral, em relação aos recursos aplicados, verificou-se um aumento real em todas as classes populacionais, no entanto esse aumento foi mais evidenciado nos municípios com mais de 100 mil habitantes.

Santos (2019), destaca que, possivelmente, gastos com caraterísticas fundamentais para classificá-los como ligados à promoção de políticas públicas de desporto e lazer acabam alocados em outras pastas, por exemplo, o desporto escolar, que pode potencializar o desenvolvimento educacional nas diversas áreas do conhecimento, pois contribui para a prática interdisciplinar dos conteúdos.

O relatório proposto por agências das Nações Unidas, em 2003, sobre as metas do esporte para o desenvolvimento e a paz no milênio, argumenta que há evidências dos impactos positivos dos programas de educação física nas escolas sobre a melhoria do desempenho acadêmico e assim, potencialização da qualidade da educação.

Adicionalmente é importante mencionar que ações integradas e coletivas, em cenário interdisciplinar, propostas por escola, família e sociedade podem potencializar a melhoria da aprendizagem e, o alcance dos indicadores sociais de forma mais significativa.

Silva Filho e Araújo (2017) comentam a mediação familiar como fator importante para ajudar o estudante a construir uma reflexão conjunta e decisiva para evitar a saída da escola antes da conclusão dos estudos. Sachsida, Loureiro e Mendonça (2004) adicionam essa importância familiar ao mostrar uma relação direta entre escolaridade do indivíduo e da mãe.

Machado e Batista (2012) complementam que a organização do conhecimento está focada em uma nova trajetória orientada pela interdisciplinaridade, deixando para trás a visão fragmentada das formas de produção e socialização do saber em diversas áreas, inclusive educação. Dessa forma, esse horizonte renovado é complementar para elevar os índices educacionais.

Machado (2009) argumenta que a prática pedagógica interdisciplinar é um processo de transformação do antigo e construção do novo, que ocorrem pela construção do saber teórico e prática em cenário reflexivo.

\section{Metodologia}

Kauark, Manhães e Medeiros (2010) argumentam que a análise quantificável que utiliza recursos e técnicas estatísticas, dentre elas a análise de regressão, caracteriza a pesquisa quantitativa. Essa pesquisa quantitativa utiliza o método indutivo para abordar o assunto. De acordo com Marconi e Lakatos (2017), esse método é mais abrangente na abordagem dos fenômenos, pois procura chegar a leis e teorias gerais partindo de constatações particulares. O objetivo da pesquisa é descritivo e segundo Gil (2008), a descrição da característica de um fenômeno ou a determinação das relações entre as variáveis estudadas representa o objetivo principal desse tipo de pesquisa.

Essa seção está dividida em duas subseções. A subseção 3.1 apresenta as fontes e as definições dos dados utilizados na pesquisa e a seção 3.2 apresenta o modelo econométrico considerado.

\subsection{Base de dados}

Nessa pesquisa, estamos interessados em observar e analisar os fatores que produzem efeitos na escolaridade, anos de estudo (média para pessoas com 25 anos ou mais de idade) no período 2002-2014 para todas as unidades federativas do Brasil, utilizando dados em painel com efeitos fixo e aleatório. O quadro 1, a seguir, indica as variáveis utilizadas, as fontes de coleta dos dados oficiais, o período e o sinal esperado nos resultados. O modelo econométrico é apresentado na subseção 3.2. 
Quadro 1 - Fontes e definições dos dados adotados.

\begin{tabular}{|c|c|c|c|c|}
\hline Variável & Período & Fonte & & $\begin{array}{l}\text { Sinal } \\
\text { esperado }\end{array}$ \\
\hline $\begin{array}{l}\text { Anos de estudo - } \\
\text { média - pessoas } 25 \\
\text { anos e mais. } \\
\quad \text { (escol) }\end{array}$ & $2002-2014$ & IPEADATA & $\begin{array}{l}\text { "Razão entre o somatório do número de anos de } \\
\text { estudo completados pelas pessoas que tem } 25 \text { ou } \\
\text { mais anos de idade e o numero de pessoas nessa } \\
\text { faixa } \\
\text { Unidade: Ano. }\end{array}$ & \\
\hline $\begin{array}{l}\text { Despesas por } \\
\text { função - esportes e } \\
\text { lazer - Estadual. } \\
\text { (esplaz). }\end{array}$ & $2002-2014$ & \begin{tabular}{l}
\multicolumn{3}{l}{ IPEADATA/Mi } \\
nistério da \\
Fazenda/ \\
Secretaria do \\
Tesouro \\
Nacional. \\
\end{tabular} & $\begin{array}{l}\text { "Estão discriminadas as despesas segundo } \\
\text { rubrica: Desportes e Lazer". Unidade: R\$ (por } \\
\text { bilhão) }\end{array}$ & Positivo \\
\hline $\begin{array}{l}\text { Domicílios - com } \\
\text { instalação adequada } \\
\text { de esgoto - pessoas } \\
\text { (sanit) }\end{array}$ & $2002-2014$ & $\begin{array}{l}\text { IPEADATA/IP } \\
\text { EA }\end{array}$ & $\begin{array}{l}\text { "Porcentagem das pessoas que vivem em } \\
\text { domicílios particulares permanentes com acesso a } \\
\text { instalações de esgoto, ou seja, que tem banheiro } \\
\text { de uso exclusivo e com escoadouro conectado à } \\
\text { rede coletora de esgoto ou pluvial ou a uma fossa } \\
\text { séptica ligada ou não a uma rede coletora". }\end{array}$ & Positivo \\
\hline $\begin{array}{l}\text { Domicílios - com } \\
\text { água potável na } \\
\text { rede geral - pessoas } \\
\text { (aguabas) }\end{array}$ & 2002-2014 & IPEADATA & $\begin{array}{l}\text { Percentual de pessoas em domicílios com } \\
\text { abastecimento de água através de rede geral com } \\
\text { canalização interna ou através de poço ou } \\
\text { nascente com canalização interna. }\end{array}$ & Positivo \\
\hline $\begin{array}{l}\text { Despesas por } \\
\text { função - saúde e } \\
\text { saneamento - } \\
\text { Estadual. } \\
\text { (sausan). }\end{array}$ & $2002-2014$ & \begin{tabular}{l}
\multicolumn{2}{l}{ IPEADATA/Mi } \\
nistério da \\
Fazenda/ \\
Secretaria do \\
Tesouro \\
Nacional. \\
\end{tabular} & $\begin{array}{l}\text { "Estão discriminadas as despesas segundo } \\
\text { rubrica: Saúde e Saneamento". Unidade: R } \$ \text { (por } \\
\text { bilhão). }\end{array}$ & Positivo \\
\hline $\begin{array}{l}\text { Frequência escolar } \\
\text { das pessoas na } \\
\text { faixa etária de } 7 \text { a } \\
14 \text { anos de idade. } \\
\text { (freql) }\end{array}$ & 2002-2014 & $\begin{array}{l}\text { IPEADATA/IP } \\
\text { EA }\end{array}$ & $\begin{array}{l}\text { "Razão entre o número de pessoas de } 7 \text { a } 14 \text { anos } \\
\text { de idade que frequentam a escola e o total de } \\
\text { pessoas nesta faixa etária". Unidade: }(\%) \text {. }\end{array}$ & Positivo \\
\hline $\begin{array}{l}\text { Coeficiente de } \\
\text { Gini. } \\
\text { (gini) }\end{array}$ & $2002-2014$ & IPEADATA & $\begin{array}{l}\text { Desigualdade de renda - Coeficiente de Gini. } \\
\text { "Mede o grau de desigualdade na distribuição da } \\
\text { renda domiciliar per capita entre os indivíduos. } \\
\text { Seu valor pode variar teoricamente desde 0, } \\
\text { quando não há desigualdade (as rendas de todos } \\
\text { os indivíduos têm o mesmo valor), até } 1 \text {, quando } \\
\text { a desigualdade é máxima (apenas um indivíduo } \\
\text { detém toda a renda da sociedade e a renda de } \\
\text { todos os outros indivíduos é nula). Série } \\
\text { calculada a partir das respostas à Pesquisa } \\
\text { Nacional por Amostra de Domicílios } \\
\text { (Pnad/IBGE)". }\end{array}$ & Negativo \\
\hline
\end{tabular}

Fonte: Autores.

O método de construção aborda dados em painel com efeito aleatório e efeito fixo. Segundo Gujarati (2019), os modelos utilizados para estudar as entidades pertencentes ao mesmo conjunto, ao longo do tempo, são denominados de modelos de regressão de dados em painel. Segundo o autor, ao assumir que para cada indivíduo, o coeficiente específico individual B1i seja fixo no tempo, tem-se o efeito fixo e, efeito aleatório caso esse coeficiente seja uma variável randomizada com valor médio de B1. Hausman (1978) define o teste para verificar qual dos dois modelos, efeito fixo ou efeito aleatório é o mais apropriando e, de acordo com o autor, ao aceitar a hipótese nula o efeito aleatório é o mais indicado e, em caso de rejeição, o efeito fixo. 


\subsection{Modelo econométrico}

Nesse trabalho, o modelo econométrico estimado relaciona a variável dependente, ou seja, a escolaridade (adotada como média de anos de estudo das pessoas com 25 anos ou mais de idade), com as variáveis explicativas, no qual a principal variável de interesse é a despesa com esporte e lazer. A equação da escolaridade do conjunto em estudo está definida a partir de dados em painel conforme enunciado abaixo.

$E_{\text {it }}=\beta x_{\text {it }}+\gamma_{t}+v_{\text {its }}$ para $i=1, \ldots, 27 ; t=1, \ldots 13$

em que $E_{\text {it }}$ é a escolaridade, medida em média de anos de estudo, da $i$-ésima Unidade da Federação no período t. A matriz contém intercepto, $x_{\text {it }}$ representa o vetor de variáveis explicativas, $v_{i t}$ é o termo aleatório e $\gamma_{t}$ visa captar efeitos específicos no tempo. Segundo a metodologia para dados em painel, define-se ainda que $v_{i t}=\alpha_{i}+u_{i t}$, no qual $\propto$ é um termo estocástico próprio das unidades. Generalizando:

$E_{\text {it }}=\beta x_{\text {it }}+\gamma_{\mathrm{t}}+\alpha_{\mathrm{i}}+u_{\text {it }}$ para $i=1, \ldots, 27 ; t=13$

Assim, $i$ representa a $i$-ésima unidade de corte transversal e $t$ o $t$-ésimo período de tempo. Se em cada unidade de corte transversal estiver presente o mesmo número de observações de séries temporais, então esse painel recebe a denominação de painel em equilíbrio.

De acordo com Wooldridge (2010), a abordagem clássica de dados em painel busca verificar se o componente individual ( $\alpha$ ) é ou não correlacionado com algum regressor. No primeiro caso, o modelo deve ser estimado por meio da aplicação de um estimador denominado de efeito fixo. No segundo caso, o mais indicado é estimar o modelo por efeito aleatório. Para se verificar qual das duas hipóteses é a que melhor se aplica, emprega-se o teste de Hausman já citado.

Segundo Wooldridge (2010) a estimação de $E_{\text {it }}$ depende das premissas que são determinadas a respeito do intercepto, dos coeficientes angulares e do termo de erros, $u_{i \text { it }}$.

Nesse estudo estamos considerando o modelo:

$E_{\text {it }}=f\left(x_{1}, x_{2}, x_{3}, x_{4}, x_{5}, x_{6}\right)$

$E_{\text {it }}=$ escolaridade, média de anos de estudo para pessoas na faixa etária de 25 anos ou mais de idade.

$x_{1}=$ despesas com esporte e lazer .

$x_{2}=$ condições sanitárias, percentual de pessoas que residem em moradias com instalação adequada de esgoto.

$x_{3}=$ abastecimento de água, pessoas que residem em domicílios com acesso à água potável.

$x_{4}=$ despesas públicas com saúde e saneamento.

$x_{5}=$ frequência escolar das pessoas na faixa etária dos 7 aos 14 anos de idade.

$x_{6}=$ desigualdade de renda, coeficiente de Gini.

$E_{\text {it }}=C_{\text {it }}+\beta_{1}$ esplaz $+\beta_{2}$ sanit $_{\text {it }}+\beta_{3}$ aguabas $_{\text {it }}+\beta_{4}$ sausan $_{\text {it }}+\beta_{5}$ freq $_{\text {it }}+\beta_{6}$ gini $_{\text {it }}+u_{\text {it }}$

com $i=1,2,3, \ldots, 27$ et $=2002,1994,1995, \ldots, 2014$. 
Os dados coletados são utilizados para a construção do modelo econométrico enunciado acima. Os dois modelos em dados em painel, efeito fixo e efeito aleatório, consideram a escolaridade como variável dependente e o restante das variáveis registradas no Quadro 1, são tomadas como variáveis explicativas. O modelo 1 traz uma abordagem em efeito aleatório e o modelo 2, em efeito fixo.

\section{Resultados e Discussão}

Os dois modelos econométricos indicados na tabela 1 apresentam os resultados empíricos do trabalho, que utiliza a variável escolaridade como dependente e a variável explicativa de interesse, ou seja, despesas com esporte e lazer.

As estimativas, apresentadas e enunciadas a seguir, utilizam dados em painel com efeitos aleatórios e efeitos fixos no período 2002 a 2014 para todas as unidades da federação do Brasil. Os resultados empíricos são revelados na tabela 1 e os respectivos comentários, logo a seguir.

Tabela 1 - Impactos sobre a escolaridade no Brasil no período 2002-2014. Dados em painel, efeito aleatório e efeito fixo.

\begin{tabular}{|c|c|c|c|c|}
\hline \multirow[t]{2}{*}{ Variável } & \multicolumn{2}{|c|}{ Modelo 1 - Efeito aleatório } & \multicolumn{2}{|c|}{ Modelo 2 - Efeito fixo } \\
\hline & Coeficiente & $P>|t|$ & Coeficiente & $P>\|t\|$ \\
\hline Esplaz & $\begin{array}{l}0,0006 * * * \\
(0,0001) \\
\end{array}$ & 0,000 & $\begin{array}{l}0,0006 * * * \\
(0,0001) \\
\end{array}$ & 0,000 \\
\hline Sanit & $\begin{array}{l}1,347 * * * \\
(0,208)\end{array}$ & 0,000 & $\begin{array}{l}1,336 * * * \\
(0,214)\end{array}$ & 0,000 \\
\hline Aguabas & $\begin{array}{l}2,737 * * * \\
(0,392)\end{array}$ & 0,000 & $\begin{array}{l}2,624 * * * \\
(0,402)\end{array}$ & 0,000 \\
\hline Sausan & $\begin{array}{l}0,107 * * * \\
(0,014)\end{array}$ & 0,000 & $\begin{array}{l}0,112 * * * \\
(0,014)\end{array}$ & 0,000 \\
\hline Freq1 & $\begin{array}{l}0,136 * * * \\
(0,020)\end{array}$ & 0,000 & $\begin{array}{l}0,138 * * * \\
(0,020)\end{array}$ & 0,000 \\
\hline Gini & $\begin{array}{l}-4,828 * * * \\
(0,781)\end{array}$ & 0,000 & $\begin{array}{l}-4,909 * * * \\
(0,794)\end{array}$ & 0,000 \\
\hline $\mathrm{C}$ & $\begin{array}{l}-7,512 * * * \\
(2,027)\end{array}$ & 0,000 & $\begin{array}{l}-7,608 * * * \\
(2,026)\end{array}$ & 0,000 \\
\hline
\end{tabular}

Entre parênteses estão os erros padrões. Nota: $(* * *)$ significante a 1\%; (**) significante a 5\%; (*) significante a 10\%. Modelo $1: 324$ observações, $R^{2}=0,5933$, Prob > F = 0,000. Modelo 2: 324 observações, Prob > F = 0,0000, $R^{2}=0,8045$. Fonte: Autores.

i) O teste de Hausman aceita a hipótese nula com uma estatística do Qui-quadrado no valor de 6,75 com 6 graus de liberdade e uma probabilidade 0,3447. Nesse contexto, o efeito aleatório é o mais indicado.

ii) A variável que representa a despesa com esporte e lazer é estatisticamente significativa nesse estudo e o sinal positivo do coeficiente mostra uma relação direta com a escolaridade, o que sugere que elevações nos montantes dessas despesas produzem impactos positivos na escolaridade.

iii) O coeficiente positivo observado na variável que representa as despesas com saúde e saneamento mostra a relação direta com a escolaridade. Verifica-se uma significância menor que $1 \%$ e o resultado sugere que políticas públicas voltadas para melhorar as condições de saneamento básico e saúde promovem o bem estar da sociedade e eleva a escolaridade.

iv) A variável utilizada para condições sanitárias, ou seja, o percentual de residências com acesso a instalação de esgoto, é estatisticamente significante ao nível de $1 \%$ e corrobora o resultado já evidenciado para saúde e saneamento.

v) A variável que retrata o acesso à água potável é estatisticamente significativa nesse estudo e a relação direta observada com a escolaridade mostra os impactos positivos em investimentos que promovam a acessibilidade à educação e ao bem essencial da vida, ou seja, a água. 
vi) A variável frequência escolar, das pessoas que estão na faixa etária dos 7 aos 14 anos de idade, é estatisticamente significante na pesquisa, e o sinal positivo do coeficiente confirma o já esperado, ou seja, que a presença à escola com constância, continuidade, dedicação e, principalmente, motivação para desenvolver os estudos ao longo dos anos é fundamental para elevar a escolaridade.

vii) Adicionalmente, políticas públicas que reduzam as desigualdades de rendas são essenciais para desenvolver o nível educacional e, conforme evidenciado nos resultados desse estudo, escolaridade e coeficiente de Gini mostram uma relação inversa, ou seja, quanto maiores os aumentos nessa desigualdade, piores serão os resultados observados nos índices que retratam os anos de estudo.

Finalizando, constatamos a confirmação da hipótese assumida nessa pesquisa, ou seja, investimentos em esporte e lazer promovem bem estar e estimulam os estudos, por conseguinte, conduzem a aumentos na escolaridade. Ademais, políticas públicas integradas que promovam condições sanitárias adequadas, investimentos em saúde e saneamento, facilidade no acesso à água potável, redução na desigualdade de renda, motivação para os estudos e investimentos em esporte e lazer potencializam um cenário de bem estar coletivo que fortalece os laços familiares e a boa vizinhança, capazes de promoverem o cenário ideal para o desenvolvimento sustentável da sociedade.

\section{Considerações Finais}

Nesse estudo utilizamos dados sobre escolaridade, ou seja, média de anos de estudo das pessoas com 25 anos ou mais de idade, considerada como variável dependente e outras adotadas como explicativas e que retratam a condição socioeconômica, frequência escolar, condições sanitárias, acesso à água potável e despesas em saúde e saneamento para testar e confirmar a hipótese de que aumentos nas despesas com esporte e lazer impactam aumentando a escolaridade no período estudado.

O estudo aborda em dois diferentes modelos com dados em painel, por efeito fixo e aleatório no período 2002-2014 com todas as unidades federativas do Brasil, e confirma a hipótese em ambos os modelos construídos, no entanto o teste de Hausman indica que dentre eles o efeito aleatório é o mais apropriado, com os resultados apresentados na seção 4.

Nessa pesquisa, as variáveis se apresentaram significativas ao nível de $1 \%$ e os coeficientes, os sinais esperados. Os resultados confirmam uma relação direta entre escolaridade e as despesas com esporte e lazer o que sugere que aumentos nesses gastos conduzem a melhores resultados em anos de estudo, ou seja, melhores índices na escolaridade.

Os resultados mostram que elevações nas despesas com saúde e saneamento, melhorias nas condições de acesso à água e instalação de esgotos produzem resultados positivos na escolaridade.

A variável frequência escolar, das pessoas que estão na faixa etária dos 7 aos 14 anos de idade, é estatisticamente significante, e a relação direta com a escolaridade corrobora para atestar os resultados já evidenciados, pois a presença à escola de forma constante e contínua, com dedicação e, principalmente, motivação para desenvolver as tarefas escolares ao longo dos anos, é fundamental para elevar a escolaridade.

Adicionalmente, escolaridade e desigualdade de renda apresentam relação inversa nessa pesquisa no período estudado, conforme esperado. Assim, sugere que a implementação de políticas públicas que reduzam essas desigualdades é fundamental para aumentar os índices do nível educacional. Finalizando, a hipótese assumida nessa pesquisa é confirmada, isto é, investimentos em esporte e lazer promovem bem estar e estimulam os estudos, por conseguinte, conduzem a aumentos na escolaridade. Complementarmente, as variáveis explicativas aqui estudadas sugerem políticas públicas integradas que potencializem um cenário de bem estar coletivo e fortaleçam os laços familiares e a boa vizinhança, capazes de promover o cenário ideal para o desenvolvimento sustentável da sociedade e da educação. 
Esse trabalho não esgota o assunto, no entanto contribui para a literatura ao propor um estudo que aborda os impactos dos gastos em esporte e lazer sobre os índices da escolaridade e, nesse trabalho, representado por média de anos de estudo. Contribui, também, ao propor a combinação das variáveis utilizadas.

Destaca-se como sugestão para trabalhos futuros, a formulação de novos modelos com outras variáveis explicativas e novas combinações que possam contribuir para ampliar e enriquecer a literatura sobre o assunto, além de promover novos estudos que conduzam à melhoria dos índices educacionais.

\section{Referências}

Abreu Souza, M. I., Taques, F. H., de Oliveira, J. D. C., \& Alencar, D. A. (2013). Relação entre a desigualdade e educação no Brasil: uma tentativa de dados em painel (1995-2009). Textos de Economia, 16(2), 111-142.

Borchers, J, \& Meiners, W. (2019). Educação e distribuição de renda: uma leitura crítica do Brasil. Anais do $22^{\circ}$ Encontro de Economia da Região Sul, 1-16.

Carli, A. A., \& Costa, L. A. (2020). Água potável e saneamento básico: o encontro necessário de dois direitos fundamentais à saúde da vida em geral. Revista de Direito e Sustentabilidade, 6(2).

Carvalho, J. I. C. (2018). Educação e saneamento básico: o quadro brasileiro e seus reflexos sobre o ensino, educação e saneamento básico. O quadro brasileiro e seus reflexos sobre o ensino. 1-388

Duarte, L. B., \& de Melo Belmiro, M. O. (2018). Utilização de dados em painel para analisar a relação entre a desigualdade de renda e educação nas regiões do Brasil. Revista Debate Econômico, 6(2), 4-20.

Feistel, R. A. B., \& Maestrelli, S. R. P. (2012). Interdisciplinaridade na formação inicial de professores: um olhar sobre as pesquisas em educação em ciências. Alexandria: Revista de Educação em Ciência e Tecnologia, 5(1), 155-176.

Gandra, J. M. D. F. V., Vieira, R. H., Rodrigues, C. T., \& de Oliveira, L. P. (2016). Impacto do saneamento básico na educação brasileira: Perspectivas de políticas públicas no setor. Revista ESPACIOS| Vol. 37 (Nº 34) Año 2016.

Gil, A. C. (2008). Métodos e técnicas de pesquisa social. (6a ed.), Editora Atlas SA.

Gujarati, D. (2019). Econometria: Princípios, teoria e aplicações práticas. Saraiva Educação SA.

Hausman, J. A. (1978). Specification tests in econometrics. Econometrica: Journal of the econometric society, 1251-1271.

Ipeadata, I. P. E. A., \& Nacionais, C. (2018). < http://www. ipeadata. gov. br/>.

Junior, A. D. P., \& da Silva, R. M. (2019). Fatores determinantes para a escolaridade na região sul do Brasil. Revista Estudo \& Debate, 26(4).

Kassouf, A. L. (1995). Saneamento e educação. Bens substitutos ou complementares. ANPEC. XXII Encontro Nacional de Economia.

Kauark, F. D. S., Manhães, F. C., \& Medeiros, C. H. (2010). Metodologia da pesquisa: um guia prático.

Kroth, D. C., \& de Oliveira Gonçalves, F. (2019). O impacto dos gastos públicos municipais sobre a qualidade da educação: uma análise de variáveis instrumentais entre 2007 e 2011. Planejamento e Políticas Públicas, (53).

Machado, M. D. M. B. C. (2009). A interdisciplinaridade na construção dos conteúdos curriculares do Curso Médico da Unimontes.

Machado, M. D. M. B. C., \& Batista, S. H. S. D. S. (2012). Interdisciplinaridade na construção dos conteúdos curriculares do curso médico. Revista Brasileira de Educação Médica, 36, 456-462.

Marconi, M. D. A., \& Lakatos, E. M. (2017). Fundamentos de metodologia científica. (8a ed.), Atlas.

Medeiros, M. C., Barbosa, R. J, \& Carvalhaes, F. (2019). Educação, desigualdade e redução da pobreza no Brasil (No. 2447). Texto para Discussão.

Moraes, D. Z, de Souza, E. C., \& do Amparo, P. A. (2009). Política educacional e representações de justiça, êxito e fracasso na escola: o exame do periódico Ideias (1988-2004). Educação Unisinos, 13(2), 152-161.

Neri, M. C. (2008). Saneamento, educação, trabalho e turismo.

Neri, M. C., Melo, L., Sacramento, S., Neri, A. L., Calcada, A. L., Scharth, P., \& Moreira, L. (2009). Trata Brasil: impactos sociais da falta de saneamento nas principais cidades brasileiras. FGV/IBRE, CPS, 120.

ONU. (2003). Esporte para o Desenvolvimento e a Paz: Em Direção à Realização das Metas de Desenvolvimento do Milênio. Relatório da Força Tarefa entre Agências das Nações Unidas sobre o Esporte para o Desenvolvimento e a Paz. Organização das Nações Unidas (ONU).

Prearo, L. C., Maraccini, M. C., \& do Carmo Romeiro, M. (2015). Fatores determinantes do índice de desenvolvimento humano dos municípios do Estado De São Paulo. Revista Brasileira de Políticas Públicas, 5(1), 132-155. 
Research, Society and Development, v. 10, n. 13, e29101321110, 2021

(CC BY 4.0) | ISSN 2525-3409 | DOI: http://dx.doi.org/10.33448/rsd-v10i13.21110

Sachsida, A., Loureiro, P. R. A., \& Mendonça, M. J. C. D. (2004). Um estudo sobre retorno em escolaridade no Brasil. Revista Brasileira de Economia, 58, 249-265.

Santos, A. G. D. (2019). Papel dos fatores políticos na decisão dos gastos com esporte e lazer nos municípios entre 2002 e 2016: evidências para as regiões Sul e Sudeste do Brasil.

Scriptore, J. S. (2016). Impactos do saneamento sobre saúde e educação: uma análise espacial. USP.

Scriptore, J. S., Azzoni, C. R., \& Menezes Filho, N. A. (2018). Os Impactos Do Saneamento Básico Sobre A Educação: Usando A Privatização Como Variável Instrumental.

Silva Filho, R. B., \& de Lima Araújo, R. M. (2017). Evasão e abandono escolar na educação básica no Brasil: fatores, causas e possíveis consequências. Educação por escrito, 8(1), 35-48.

Silva, H. M. G. D. (2015). A condição socioeconômica e cultural e o acesso à educação básica. Desafios Contemporâneos da educação. Cultura Acadêmica, 177-191.

Silva, V. A., \& Esperidião, F. (2017). Saneamento básico e seus impactos na mortalidade infantil e no desenvolvimento econômico da região Nordeste. Scientia Plena, 13(10).

Sousa, T. R. V., \& Leite Filho, P. A. M. (2008). Análise por dados em painel do status de saúde no Nordeste Brasileiro. Revista de Saúde Pública, 42, 796804.

Varela, A. W. P., Noronha Filho, F. Áureo, Mesquita, K. F. C., Sousa, P. H. C., Neves de Souza, A. J, \& Santos, M. de L. S. (2020). Saneamento básico em municípios da região Amazônica (Brasil). Research, Society and Development, 9(3), e177932570.

Wooldridge, J. M. (2010). Introdução à econometria: uma abordagem moderna. Cengage Learning. 